\title{
DUAL OF THE AUSLANDER-BRIDGER FORMULA AND GF-PERFECTNESS
}

\author{
PARVIZ SAHANDI and TIRDAD SHARIF*
}

\begin{abstract}
Ext-finite modules were introduced and studied by Enochs and Jenda. We prove under some conditions that the depth of a local ring is equal to the sum of the Gorenstein injective dimension and Tor-depth of an Ext-finite module of finite Gorenstein injective dimension. Let $(R, \mathrm{~m})$ be a local ring. We say that an $R$-module $M$ with $\operatorname{dim}_{R} M=n$ is a Grothendieck module if the $n$-th local cohomology module of $M$ with respect to $\mathrm{m}, \mathrm{H}_{\mathfrak{m}}^{n}(M)$, is non-zero. We prove the Bass formula for this kind of modules of finite Gorenstein injective dimension and of maximal Krull dimension. These results are dual versions of the Auslander-Bridger formula for the Gorenstein dimension. We also introduce GF-perfect modules as an extension of quasi-perfect modules introduced by Foxby.
\end{abstract}

\section{Introduction}

Throughout this paper all rings are commutative and Noetherian with nonzero identity. In [8] and [10] Enochs and Jenda introduced and studied mock finite Gorenstein injective modules. As an extension they introduced and studied the Ext-finite modules of finite Gorenstein injective dimension in [11]. We recall that an $R$-module $M$ is called Ext-finit if $\operatorname{Ext}_{R}^{i}(N, M)$ is finite (i.e. finitely generated) for each finite $R$-module $N$ and for $i \geq 1$. Therefore, every finite $R$-module $M$ is Ext-finite and it is also easy to see that every cosyzygy of an Ext-finite module is also Ext-finite [11, (4.7)]. In section 2, following Enochs and Jenda in [11] we prove a dual result for the Auslander-Bridger formula [1] for Ext-finite modules of finite Gorenstein injective dimension. Our approach to obtain a dual result is fundamentally different from the method of Enochs and Jenda in [11]. In this direction, we show that an $R$-module $M$ with finite Gorenstein injective dimension (Gid) has a surjective precover $N$, such that $\operatorname{Gid}_{R} M=\operatorname{id}_{R} N$, with respect to the class $\mathscr{I}_{0}$ of modules of finite injective dimension. We call the $R$-module $N$ an $\mathscr{I}_{0}$-precover of $M$. Viewing this, we introduce GI-syzygy modules for modules of finite Gorenstein injective dimension, see (2.3). These objects play an important role to prove one of our main results in Section 2, see Theorem (2.4).

\footnotetext{
* The second author is supported by a grant from IPM (No. 83130311)
}

Received January 19, 2006. 
Let $(R, \mathfrak{m})$ be a local ring, and let $M$ be an $R$-module of Krull dimension $n$. We say that $M$ is a Grothendieck module, if the $n$-th local cohomology module of $M$ with respect to $\mathfrak{m}, \mathrm{H}_{\mathfrak{m}}^{n}(M)$, is non-zero. From the non-vanishing Theorem of Grothendieck [4, (6.1.4)] it follows that all finite modules are Grothendieck modules. In [20] R. Sazeedeh used local cohomology to study the Gorenstein injective modules over Gorenstein rings. By developing his method over arbitrary local rings, we prove a dual result for the AuslanderBridger formula, see Theorem (2.7).

In Section 3 we introduce and study a new invariant for an $R$-module $M$ denoted by F-grade ${ }_{R} M$ which is, an extension of the usual notion of $\operatorname{grade}_{R} M$ introduced by Rees in [18]. An $R$-module $M$ of finite Gorenstein flat dimension (Gfd) is called GF-perfect if F- $\operatorname{grade}_{R} M=\operatorname{Gfd}_{R} M$. This concept generalizes the notion of quasi-perfectness introduced by Foxby in [13]. We prove that for GF-perfect modules of finite depth over Cohen-Macaulay local rings we have $\operatorname{dim}_{R} M=\operatorname{depth}_{R} M$. In Corollary (3.9) we state an Auslander-Bridger formula for GF-perfect modules of finite depth over Cohen-Macaulay rings. We also investigate the behavior of GF-perfect modules under the first fundamental change of rings, see Corollary (3.12).

Throughout this paper, we use the following notions:

(1) The Gorenstein injective modules were introduced by Enochs and Jenda in [8]. An $R$-module $M$ is said to be Gorenstein injective if and only if there is an exact sequence

$$
\cdots \longrightarrow E^{-1} \longrightarrow E^{0} \longrightarrow E^{1} \longrightarrow \cdots
$$

of injective $R$-modules such that $M=\operatorname{ker}\left(E^{0} \longrightarrow E^{1}\right)$, and such that for any injective $R$-module $E, \operatorname{Hom}_{R}(E,-)$ leaves the complex above exact. The above complex is known as complete injective resolution.

(2) The Gorenstein flat modules were introduced by Enochs, Jenda, and Torrecillas in [9]. An $R$-module $M$ is said to be Gorenstein flat if and only if there is an exact sequence

$$
\cdots \longrightarrow F^{-1} \longrightarrow F^{0} \longrightarrow F^{1} \longrightarrow \cdots
$$

of flat $R$-modules such that $M=\operatorname{ker}\left(F^{0} \longrightarrow F^{1}\right)$, and such that for any injective $R$-module $I, I \otimes_{R}$ - leaves the complex above exact. The above complex is known as complete flat resolution.

(3) The Gorenstein flat dimension (Gfd) and the Gorenstein injective dimension (Gid), respectively are defined by using the Gorenstein flat modules and the Gorenstein injective modules, by a similar fashion as the flat dimension (fd) and the injective dimension (id), respectively are defined. 
(4) Let $\mathscr{X}$ be a class of $R$-modules for some ring $R$. If $\phi: X \longrightarrow M$ is linear where $X \in \mathscr{X}$ and $M$ is an $R$-module, then $\phi: X \longrightarrow M$ is called an $\mathscr{X}$-precover of $M$ if

$$
\operatorname{Hom}_{R}(Y, X) \longrightarrow \operatorname{Hom}_{R}(Y, M) \longrightarrow 0
$$

is exact for all $Y \in \mathscr{X}$.

(5) $\mathscr{P}_{0}=\{M \mid M$ is an R-module of finite projective dimension $\}$.

(6) $\mathscr{I}_{0}=\{M \mid M$ is an R-module of finite injective dimension $\}$.

\section{Dual of the Auslander-Bridger formula}

In this section we prove a dual of the Auslander-Bridger formula in Theorems (2.4) and (2.7). In order to prove the theorems we need two lemmas. The following lemma gives a characterization for Cohen-Macaulay local rings.

Lemma 2.1. Let $(R, \mathfrak{m}, k)$ be a local ring and let $N$ be an Ext-finite $R$-module of finite injective dimension and of finite depth. Then

$$
\operatorname{id}_{R} N=\sup \left\{i \mid \operatorname{Ext}_{R}^{i}(T, N) \neq 0 \text { for some } T \in \mathscr{P}_{0} \text { with } \ell_{R}(T)<\infty\right\},
$$

if and only if $R$ is a Cohen-Macaulay ring.

Proof. First of all suppose that the equality holds. Then there is an $R$ module $T$ of finite length and of finite projective dimension. Hence $R$ is CohenMacaulay by the Intersection Theorem cf. [19]. Conversely, suppose that $R$ is a Cohen-Macaulay ring. By [21, (1.4)] $n=\operatorname{id}_{R} N=\sup \left\{i \mid \operatorname{Ext}_{R}^{i}(k, N) \neq 0\right\}$. Then $\operatorname{Ext}_{R}^{n}(k, N) \neq 0$. Let $x_{1}, \ldots, x_{t}$ be a maximal $R$-sequence in $\mathfrak{m}$. Since $R$ is Cohen-Macaulay $\mathfrak{m} \in \operatorname{Ass}\left(R /\left(x_{1}, \ldots, x_{t}\right)\right)$. Let $T=R /\left(x_{1}, \ldots, x_{t}\right)$. So we have the exact sequence $0 \longrightarrow k \longrightarrow T \longrightarrow L \longrightarrow 0$, which induces the exact sequence

$$
\operatorname{Ext}_{R}^{n}(T, N) \longrightarrow \operatorname{Ext}_{R}^{n}(k, N) \longrightarrow 0 .
$$

So that $\operatorname{Ext}_{R}^{n}(T, N) \neq 0$, and this completes the proof.

Lemma 2.2. Let $R$ be a ring and let $M$ be an $R$-module with $\operatorname{Gid}_{R} M<\infty$. Then there is a surjective $\mathscr{I}_{0}$-precover $\varphi: N \longrightarrow M$ such that $\operatorname{id}_{R} N=$ $\operatorname{Gid}_{R} M$ and $\operatorname{ker} \varphi$ is a Gorenstein injective R-module.

Proof. We use an induction argument on $g=\operatorname{Gid}_{R} M$. If $g=0$, then $M$ is Gorenstein injective. So by definition of the Gorenstein injective modules, there is the exact sequence

$$
0 \longrightarrow H \longrightarrow I \longrightarrow M \longrightarrow 0,
$$


in which $I$ is injective and $H$ is Gorenstein injective. By [8, Proposition (2.4)] it is clear that $I$ is an $\mathscr{I}_{0}$-precover of $M$. Now let $g \geqslant 1$. From [16, (2.15)] there is a Gorenstein injective module $G$ and an $R$-module $L$ with $\operatorname{id}_{R} L=g-1$ such that the following sequence is exact

$$
0 \longrightarrow M \longrightarrow G \longrightarrow L \longrightarrow 0 \text {. }
$$

Since $G$ is Gorenstein injective we have the following pullback diagram

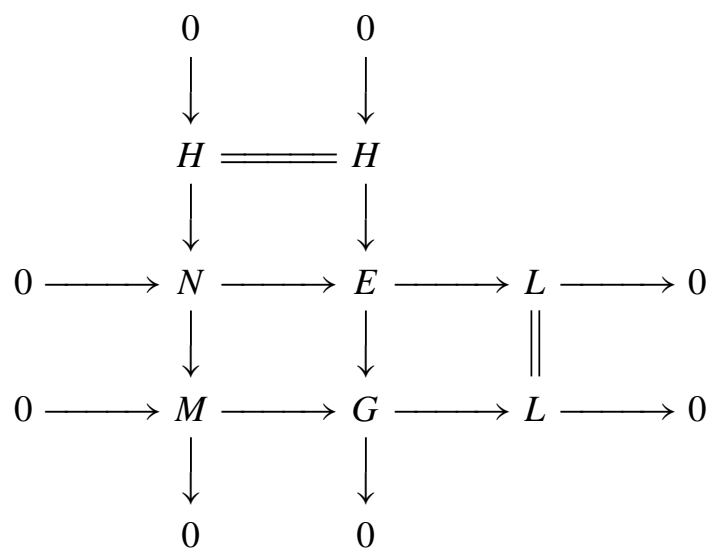

where $H$ is Gorenstein injective module and $\mathrm{id}_{R} N=g$. Now from [8, Proposition (2.4)] it follows that in the exact sequence

$$
0 \longrightarrow H \longrightarrow N \longrightarrow M \longrightarrow 0
$$

$N$ is an $\mathscr{I}_{0}$-precover of $M$ such that $\operatorname{id}_{R} N=\operatorname{Gid}_{R} M$.

Let $M$ be an $R$-module with $\operatorname{Gid}_{R} M<\infty$. From the above lemma we have the exact sequence

$$
0 \longrightarrow H_{1} \longrightarrow N \longrightarrow M \longrightarrow 0
$$

where $H_{1}$ is Gorenstein injective. From the definition of Gorenstein injective modules there is an injective $R$-module $E_{1}$ and a Gorenstein injective module $\mathrm{H}_{2}$ such that the following sequence is exact.

$$
0 \longrightarrow H_{2} \longrightarrow E_{1} \longrightarrow H_{1} \longrightarrow 0 \text {. }
$$

By continuing in the same manner we can find Gorenstein injective modules $H_{i}$ and injective modules $E_{i}$ for $i \geqslant 1$ such that the sequence

$$
\cdots \longrightarrow E_{2} \longrightarrow E_{1} \longrightarrow N \longrightarrow M \longrightarrow 0
$$


is exact. It is clear that $\operatorname{id}_{R} M<\infty$ if and only if the above sequence is finite.

DEFINITION 2.3. In the above construction we call the $R$-modules $H_{i}$ for $i \geqslant 1$ the $i$-th $G I$-syzygy module of $M$.

Now we are in the position of proving the first main result of this section. Recall that

$$
\operatorname{Tor}_{-\operatorname{depth}} M=\inf \left\{i \mid \operatorname{Tor}_{i}^{R}(k, M) \neq 0\right\} .
$$

It is shown in $[15,(14.17)]$ that Tor-depth ${ }_{R} M$ is finite if and only if $\operatorname{depth}_{R} M$ is finite.

Theorem 2.4. Let $(R, \mathfrak{m}, k)$ be a Cohen-Macaulay local ring and let $M$ be an Ext-finite $R$-module of infinite injective dimension. If $\operatorname{Gid}_{R} M<\infty$ and $M$ has an Ext-finite GI-syzygy module, then

$$
\operatorname{Gid}_{R} M+\text { Tor-depth }_{R} M=\operatorname{depth} R \text {. }
$$

Proof. Let $g=\operatorname{Gid}_{R} M$. If $g=0$, then [11, (4.1)] gives the result. Now suppose that $g \geqslant 1$, so $M$ has an $\mathscr{I}_{0}$-precover $N$, with $\operatorname{id}_{R} N=g$, and such that in the exact sequence

$$
0 \longrightarrow H \longrightarrow N \longrightarrow M \longrightarrow 0
$$

$H$ is a Gorenstein injective module with $\operatorname{id}_{R} H=\infty$. By definition of $G I$ syzygy modules of $M$, it is easy to see that $H$ is an Ext-finite module. So by [11, (4.1)] Tor-depth ${ }_{R} H=\operatorname{depth} R=\operatorname{dim} R=d$. If Tor-depth ${ }_{R} M=\infty$, from the long exact sequence of homologies we get that Tor-depth ${ }_{R} N=$ Tor-depth $_{R} H=d$. Since $M$ and $H$ are Ext-finite modules, then so is $N$. Now from $[21,(1.6)]$ it follows that $\operatorname{id}_{R} N=0$. This yields that $g=0$, which is a contradiction, hence Tor-depth ${ }_{R} M<\infty$. On the other hand, since Tor-depth $_{R} H=d$, we get $\operatorname{depth}_{R} H=0$ by [15, (14.18)], and this yields that $\operatorname{depth}_{R} N=0$. So we obtain that Tor-depth ${ }_{R} N<\infty$ by [15, (14.18)] again. Since $R$ is a Cohen-Macaulay ring, from Lemma (2.1) we get that $\operatorname{Ext}_{R}^{g}(T, N) \neq 0$ for some $T \in \mathscr{P}_{0}(R)$ of finite length. From the long exact sequence induced by $(*)$ and [8, Proposition $(2.4)]$ we find that there is an exact sequence as follows

$$
0=\mathrm{Ext}_{R}^{g}(T, H) \longrightarrow \operatorname{Ext}_{R}^{g}(T, N) \longrightarrow \operatorname{Ext}_{R}^{g}(T, M) \longrightarrow 0 .
$$

Therefore

$$
\operatorname{Gid}_{R} M=\sup \left\{i \mid \operatorname{Ext}_{R}^{i}(T, M) \neq 0 \text { for some } T \in \mathscr{P}_{0} \text { with } \ell_{R}(T)<\infty\right\} \text {, }
$$


and so we find that

$\operatorname{Gid}_{R} M$

$=\sup \left\{i \mid \operatorname{Hom}_{R}\left(\operatorname{Ext}_{R}^{i}(T, M), E(k)\right) \neq 0\right.$ for some $T \in \mathscr{P}_{0}$ with $\left.\ell_{R}(T)<\infty\right\}$

$=\sup \left\{i \mid \operatorname{Tor}_{i}^{R}(T, \operatorname{Hom}(M, E(k))) \neq 0\right.$ for some $T \in \mathscr{P}_{0}$ with $\left.\ell_{R}(T)<\infty\right\}$.

On the other hand, $\operatorname{since}_{\operatorname{depth}} T=0$ and $\operatorname{depth}_{R} \operatorname{Hom}_{R}(M, E(k))=$ Tor-depth ${ }_{R} M$, from [22, (2.3)], it follows that the right side of the second equality is depth $R-$ Tor-depth $_{R} M$ as desired.

Now it is natural to ask the following question:

QuESTION 2.5. How can we decide about the Ext-finiteness (mock finiteness) of $N$ in Lemma 2.2, when $M$ is an Ext-finite (mock finite) module?

Note that a consequence of an affirmative answer to our question gives a dual result for the Auslander-Bridger formula as follows:

Let $(R, \mathfrak{m}, k)$ be a Cohen-Macaulay local ring and let $M$ be a mock finite $\mathrm{R}$-module with $\operatorname{Gid}_{R} M<\infty$ and of infinite injective dimension. Then

$$
\operatorname{Gid}_{R} M+\text { Tor-depth }_{R} M=\text { depth } R .
$$

In the rest of this section we introduce a class of modules called Grothendieck modules. We find a dual result for the Auslander-Bridger formula for this kind of modules of maximal dimension.

Definition 2.6. An $R$-module $M$ of Krull dimension $n$, is said to be a Grothendieck module if $\mathrm{H}_{\mathfrak{m}}^{n}(M) \neq 0$.

The following result is analogous to a classical result due to $\mathrm{H}$. Bass [3]. In [23] Takahashi proved the following theorem for finite modules, under the additional assumption that the base ring admits a dualizing complex. In [25] Yassemi, proved Takahashi's result, without assuming that the ring admits a dualizing complex.

THeOREM 2.7. Let $(R, \mathfrak{m})$ be a local ring and let $M$ be a Grothendieck module with $\operatorname{Gid}_{R} M<\infty$. If $\operatorname{dim} M=\operatorname{dim} R$, then $R$ is a Cohen-Macaulay ring and $\operatorname{Gid}_{R} M=$ depth $R$.

Proof. Let $g=\operatorname{Gid}_{R} M$ and let $n=\operatorname{dim} M$. By a similar argument to that of $[20,(3.1)]$ it is easy to see that $\mathrm{H}_{\mathfrak{m}}^{i}(H)=0$ when $\operatorname{Gid}_{R} H=0$ and $i>0$. Now from Lemma (2.2) it is clear that for $i>0, \mathrm{H}_{\mathfrak{m}}^{i}(M)=\mathrm{H}_{\mathfrak{m}}^{i}(N)$ when $N$ is an $\mathscr{I}_{0}$-precover of $M$ such that $\operatorname{id}_{R} N=g$. It is easy to see that in this case $\mathrm{H}_{\mathfrak{m}}^{i}(N)=0$ for $i>g$. On the other hand, since $\operatorname{dim} N=\operatorname{dim} M, N$ is a 
Grothendieck module too. Therefore $n \leq \operatorname{id}_{R} N$. Now we have the following (in)equalities:

$$
n=\operatorname{dim} R \leq \operatorname{id}_{R} N=\operatorname{depth} R_{\mathfrak{p}}-\text { Tor-depth } R_{R_{\mathfrak{p}}} N_{\mathfrak{p}} \leq \text { ht } \mathfrak{p}
$$

in which the second equality holds by $[6,(3.1)]$, so we obtain $\mathfrak{p}=\mathfrak{m}$. This ends our proof.

Recall from [5] that an $R$-module $M$ is said to be have rank $r$, if $M_{\mathfrak{p}}$ is a free $R_{\mathfrak{p}}$-module of rank $r$, for all prime ideals $\mathfrak{p} \in \operatorname{Ass}(R)$. It is clear that, finite modules with positive rank are of maximal Krull dimension.

COROLlary 2.8. If $M$ is a finite module of positive rank with finite Gorenstein injective dimension, then $R$ is a Cohen-Macaulay ring.

It is interesting to know that there is a large class of non-finite modules satisfying both conditions of Theorems 2.4 and 2.7.

Example 2.9. Let $(R, \mathfrak{m}, k)$ be an $n$-Gorenstein local ring which is not regular, and let $L$ be an $R$-module with $\ell_{R}(L)<\infty$ and $\operatorname{id}_{R} L=\infty$. Let $E$. be the minimal injective resolution of $L$. Therefore, each term of $E$, is direct sum of finitely many copies of $E(k)$, the injective envelope of $k$. So all terms of $E$ are m-torsion, in the sense of [4]. Let $H$ be the $r$-th cosyzygy of this resolution for $r \geqslant n$. By [11, (4.2) and (4.7)] $H$ is Gorenstein injective and Ext-finite. So, by $[8,(6.5)] H$ is a mock finite module. Viewing $[8,(6.6)]$ we get that, the first $G I$-syzygy of $H$ is mock finite too. Set $M=H \oplus R$, now it is clear that $M$ is an Ext-finite, Grothendieck $R$-module with $\operatorname{dim} M=\operatorname{dim} R$.

\section{GF-Perfect modules}

Let $M$ be a finite $R$-module, the notion grade ${ }_{R} M$ was defined by Rees as the least integer $\ell \geqslant 0$ such that $\operatorname{Ext}_{R}^{\ell}(M, R) \neq 0$. In [18] Rees proved that the grade $_{R} M$ is the maximum lengths of $R$-regular elements in $\operatorname{Ann}_{R}(M)$. It is easy to see that $\operatorname{grade}_{R} M$ is the least integer $\ell \geqslant 0$ such that $\operatorname{Ext}_{R}^{\ell}(M, P) \neq 0$ for some projective $R$-module $P$. When $M$ is a non-finite $R$-module there is not any extension of this important invariant, however in any extension of grade, a homological view is useful. In this section for an arbitrary $R$-module $M$ we introduce a new invariant denoted by F-grade ${ }_{R} M$, such that when $M$ is finite then F-grade ${ }_{R} M=\operatorname{grade}_{R} M$. One important concept closely related to the grade of modules is perfectness. A finite $R$-module $M$ with $\operatorname{pd}_{R} M<\infty$ is said to be perfect if grade $_{R} M=\operatorname{pd}_{R} M$. This concept was generalized by Foxby in [13] where he defined quasi-perfect modules. A finite $R$-module $M$ with G- $\operatorname{dim}_{R} M<\infty$ is said to be quasi-perfect if $\operatorname{grade}_{R} M=\mathrm{G}-\operatorname{dim}_{R} M$ (in which G- $\operatorname{dim}_{R} M$ is Gorenstein dimension of $M$ introduced by Auslander and 
Bridger in [1]). The perfect and quasi-perfect modules over Cohen-Macaulay local rings are Cohen-Macaulay modules, see [5] and [13], respectively. In this section a generalization of this fact over Cohen-Macaulay local rings is proved in Theorem (3.7) below.

Definition 3.1. Let $M$ be an $R$-module. The Flat grade of $M$ is denoted by F-grade $_{R} M$, and it is defined by the following formula

F-grade ${ }_{R} M=\inf \left\{i \mid \operatorname{Ext}_{R}^{i}(M, F) \neq 0\right.$ for some flat $R$-module $\left.F\right\}$.

By definition it is clear that F-grade ${ }_{R} M \leqslant \operatorname{grade}_{R} M$.

Remark 3.2. Let $M$ be a finite $R$-module and suppose that $\mathrm{F}$-grade ${ }_{R} M=$ $\ell$. Then there is a flat $R$-module $F$ such that $\operatorname{Ext}_{R}^{\ell}(M, F) \neq 0$. Since $M$ is finite, $\operatorname{Ext}_{R}^{\ell}(M, F) \simeq \operatorname{Ext}_{R}^{\ell}(M, R) \otimes_{R} F$ and so $\operatorname{Ext}_{R}^{\ell}(M, R) \neq 0$. Therefore, $\operatorname{grade}_{R} M \leqslant \ell$. Now we have F-grade ${ }_{R} M=\operatorname{grade}_{R} M$.

It is not difficult to see that $\mathrm{F}$ - $\operatorname{grade}_{R} M \leqslant \operatorname{Gfd}_{R} M$, it is also a trivial consequence of the following proposition. Recall from [24, Definition (3.1.1)] that an $R$-module $C$ is called cotorsion, if for all flat $R$-modules $F$, $\operatorname{Ext}_{R}^{1}(F, C)=$ 0 . The following proposition shows that F-grade and Gfd can be computed via cotorsion flat modules.

Proposition 3.3. Let $R$ be a ring and let $M$ be an $R$-module. Then

F-grade $_{R} M=\inf \left\{i \mid \operatorname{Ext}_{R}^{i}(M, F) \neq 0\right.$ for some cotorsion flat $R$-module $\left.F\right\}$, and if $\operatorname{Gfd}_{R} M<\infty$, then

$\operatorname{Gfd}_{R} M=\sup \left\{i \mid \operatorname{Ext}_{R}^{i}(M, F) \neq 0\right.$ for some cotorsion flat $R$-module $\left.F\right\}$.

Proof. If F-grade ${ }_{R} M=\infty$, then $\operatorname{Ext}_{R}^{i}(M, F)=0$ for all flat $R$-modules, thus the right side is infinity too. Let F-grade ${ }_{R} M=n$, therefore $\operatorname{Ext}_{R}^{n}(M, F)$ $\neq 0$ for some flat $R$-module $F$. Now let $Q$ be the pure injective envelope of $F$ cf. [24]. By [24, (3.1.6)] $Q / F=H$ is flat, thus $\operatorname{Ext}_{R}^{i}(M, H)=0$ for $i<n$. On the other hand the exact sequence $0 \longrightarrow F \longrightarrow Q \longrightarrow H \longrightarrow 0$ gives rise to an injection

$$
0 \longrightarrow \operatorname{Ext}_{R}^{n}(M, F) \longrightarrow \operatorname{Ext}_{R}^{n}(M, Q) \longrightarrow \cdots .
$$

Therefore $\operatorname{Ext}_{R}^{n}(M, Q) \neq 0$. Keep in mind that pure injective modules are cotorsion.

Now let $g=\operatorname{Gfd}_{R} M<\infty$. From [16] we can find an injective module $J$ such that $\operatorname{Tor}_{g}^{R}(M, J) \neq 0$. Therefore $\operatorname{Hom}_{R}\left(\operatorname{Tor}_{g}^{R}(M, J), Q\right) \neq 0$ for a 
faithfully injective $R$-module $Q$. This yields that $\operatorname{Ext}_{R}^{g}\left(M, \operatorname{Hom}_{R}(J, Q)\right) \neq 0$. By setting $F=\operatorname{Hom}_{R}(J, Q)$ and considering the simple fact that $F$ is a flat cotorsion $R$-module, we see that the right side is greater than or equal to $g$. On the other hand, let $F$ be a cotorsion flat $R$-module. Therefore, from [12, (2.3)] it follows that there is a flat $R$-module $H$, and injective $R$-modules $J_{1}$ and $J_{2}$ such that $F \oplus H=\operatorname{Hom}_{R}\left(J_{1}, J_{2}\right)$. Let for some $i>g$, $\operatorname{Ext}_{R}^{i}(M, F) \neq$ 0 . Hence $\operatorname{Ext}_{R}^{i}\left(M, \operatorname{Hom}_{R}\left(J_{1}, J_{2}\right)\right)=\operatorname{Hom}_{R}\left(\operatorname{Tor}_{i}^{R}\left(M, J_{1}\right), J_{2}\right) \neq 0$ and so $\operatorname{Tor}_{i}^{R}\left(M, J_{1}\right) \neq 0$. From this and [16] we have $\operatorname{Gfd}_{R} M>g$, which is a contradiction.

Definition 3.4. Let $M$ be an $R$-module with $\operatorname{Gfd}_{R} M<\infty$. We call $M$ Gorenstein flat perfect (GF-perfect for short) if F- $\operatorname{grade}_{R} M=\operatorname{Gfd}_{R} M$.

Note that a finite $R$-module $M$ is quasi-perfect if and only if it is GFperfect, because G-dim ${ }_{R} M=\operatorname{Gfd}_{R} M$ by [9] and F-grade ${ }_{R} M=\operatorname{grade}_{R} M$ by Remark 3.2.

Lemma 3.5. Let $R$ be a ring and let $M$ be a GF-perfect $R$-module, then for $\mathfrak{p} \in \operatorname{Supp}_{R} M, M_{\mathfrak{p}}$ is a GF-perfect $R_{\mathfrak{p}}$-module.

Proof. Since $\operatorname{Gfd}_{R} M<\infty$ it is easy to see that $\operatorname{Gfd}_{R_{\mathfrak{p}}} M_{\mathfrak{p}}<\infty$. Thus we have F-grade $R_{R_{\mathfrak{p}}} M_{\mathfrak{p}} \leqslant \operatorname{Gfd}_{R_{\mathfrak{p}}} M_{\mathfrak{p}}<\infty$. Set F-grade $R_{\mathfrak{p}} M_{\mathfrak{p}}=n$. So there is a flat $R_{\mathfrak{p}}$-module $Q$ such that $\operatorname{Ext}_{R_{\mathfrak{p}}}^{n}\left(M_{\mathfrak{p}}, Q\right) \neq 0$. Consider an $R$-projective resolution $P$. $\longrightarrow M$, hence $P_{\mathfrak{p p}}$ is a projective resolution for $M_{\mathfrak{p}}$. Now we have the following equalities

$$
\begin{aligned}
0 \neq \operatorname{Ext}_{R_{\mathfrak{p}}}^{n}\left(M_{\mathfrak{p}}, Q\right) & =\mathrm{H}^{n}\left(\operatorname{Hom}_{R_{\mathfrak{p}}}\left(P_{. \mathfrak{p}}, Q\right)\right) \\
& =\mathrm{H}^{n}\left(\operatorname{Hom}_{R}\left(P_{.}, Q\right)\right)=\operatorname{Ext}_{R}^{n}(M, Q)
\end{aligned}
$$

Since $Q$ is also flat as an $R$-module, then F-grade ${ }_{R} M \leqslant n$. Hence we have the following chain of inequalities

$$
\text { F-grade }{ }_{R} M \leqslant \text { F-grade } R_{\mathfrak{p}} M_{\mathfrak{p}} \leqslant \operatorname{Gfd}_{R_{\mathfrak{p}}} M_{\mathfrak{p}} \leqslant \operatorname{Gfd}_{R} M .
$$

Since M is GF-perfect so $\operatorname{Gfd}_{R} M=$ F-grade ${ }_{R} M$, therefore $M_{p}$ is also GFperfect $R_{\mathfrak{p}}$-module.

The following lemma is well known [14]. We include a proof here for completeness.

Lemma 3.6. Let $(R, \mathfrak{m})$ be a local ring with $\mathrm{cmd} R=\operatorname{dim} R-\operatorname{depth} R \leqslant 1$. If $\mathfrak{p}, \mathfrak{q} \in \operatorname{Spec}(R)$ and $\mathfrak{p} \subseteq \mathfrak{q}$, then depth $R_{\mathfrak{p}} \leqslant \operatorname{depth} R_{\mathfrak{q}}$.

Proof. If $\mathrm{cmd} R=0$ there is nothing to prove. Now we can assume cmd $R=1$. We will induct on $d=\operatorname{dim} R$. If $d=0$ it is trivial. Assume $d>0$, 
and let $\mathfrak{p} \subseteq \mathfrak{q}$. If $\mathfrak{q}=\mathfrak{m}$, viewing [2], we get that depth $R_{\mathfrak{p}} \leqslant \operatorname{dim} R-1=$ depth $R_{\mathfrak{q}}$. Now let $\mathfrak{q} \neq \mathrm{m}$, since $\operatorname{dim} R_{\mathfrak{q}} \supsetneqq d$ and $\mathrm{cmd} R_{\mathfrak{q}} \leqslant \mathrm{cmd} R \leqslant 1$, by the induction hypothesis we have depth $R_{\mathfrak{p}}=\operatorname{depth}\left(R_{\mathfrak{q}}\right)_{\mathfrak{p} R_{\mathfrak{q}}} \leqslant \operatorname{depth} R_{\mathfrak{q}}$.

In the following theorem we generalized, results of Rees and Foxby in [18] and [13]. For the proof we need to recall the definition of the invariant Rfd which is called Large Restricted flat dimension, introduced and studied by Christensen, Foxby and Frankild in [7]. It is defined by the formula

$\operatorname{Rfd}_{R} M=\sup \left\{i \mid \operatorname{Tor}_{i}^{R}(L, M) \neq 0\right.$ for some $R$-module $L$ with $\left.\mathrm{fd}_{R} L<\infty\right\}$.

This number is finite, as long as $M$ is nonzero and the Krull dimension of $\mathrm{R}$ is finite; see [7, (2.2)]. They proved that, see [7, (2.4)]

$$
\operatorname{Rfd}_{R} M=\sup \left\{\operatorname{depth} R_{\mathfrak{p}}-\operatorname{depth}_{R_{\mathfrak{p}}} M_{\mathfrak{p}} \mid \mathfrak{p} \in \operatorname{Spec}(R)\right\} .
$$

THeOREM 3.7. Let $(R, \mathrm{~m})$ be a local ring such that $\mathrm{cmd} R \leqslant 1$. Then for any GF-perfect $R$-module $M$ of finite depth we have:

$$
\operatorname{depth} R-\operatorname{depth}_{R} M \leqslant \text { F-grade }{ }_{R} M \leqslant \operatorname{dim} R-\operatorname{dim} M .
$$

In particular, if $R$ is a Cohen-Macaulay ring, then $\operatorname{depth}_{R} M=\operatorname{dim} M$.

Proof. First of all we show that for each $\mathfrak{p} \in \operatorname{Ass}_{R}(M), \mathrm{F}-\operatorname{grade}_{R} M=$ depth $R_{\mathfrak{p}}$. Choose $\mathfrak{p} \in \operatorname{Ass}_{R}(M)$. Thus depth $R_{R_{\mathfrak{p}}} M_{\mathfrak{p}}=0$. Since $\operatorname{Gfd}_{R} M<\infty$ therefore by [7] and [16, (3.19)] we have

$$
\operatorname{depth} R_{\mathfrak{p}}=\operatorname{depth} R_{\mathfrak{p}}-\operatorname{depth}_{R_{\mathfrak{p}}} M_{\mathfrak{p}} \leqslant \operatorname{Gfd}_{R} M .
$$

Since $\operatorname{Gfd}_{R_{\mathfrak{p}}} M_{\mathfrak{p}}<\infty$, there is by [16, (3.19)] a prime ideal $\mathfrak{q} \subseteq \mathfrak{p}$ such that

$$
\operatorname{Gfd}_{R_{\mathfrak{p}}} M_{\mathfrak{p}}=\operatorname{depth} R_{\mathfrak{q}}-\operatorname{depth}_{R_{\mathfrak{q}}} M_{\mathfrak{q}} .
$$

Hence by noting Lemma (3.6) we have:

$$
\operatorname{Gfd}_{R_{\mathfrak{p}}} M_{\mathfrak{p}} \leqslant \operatorname{depth} R_{\mathfrak{p}}-\operatorname{depth}_{R_{\mathfrak{q}}} M_{\mathfrak{q}} \leqslant \operatorname{Gfd}_{R} M-\operatorname{depth}_{R_{\mathfrak{q}}} M_{\mathfrak{q}} .
$$

By Lemma (3.5) $M_{\mathfrak{p}}$ is GF-perfect as $R_{\mathfrak{p}}$-module and Gfd $M=\operatorname{Gfd}_{R_{\mathfrak{p}}} M_{\mathfrak{p}}$. Hence depth $R_{\mathfrak{q}} M_{\mathfrak{q}}=0$ and

$$
\operatorname{depth} R_{\mathfrak{p}} \leqslant \operatorname{Gfd}_{R} M=\operatorname{Gfd}_{R_{\mathfrak{p}}} M_{\mathfrak{p}} \leqslant \operatorname{depth} R_{\mathfrak{p}} .
$$

Now our first claim is proved.

Choose $\mathfrak{p} \in \operatorname{Ass}_{R}(M)$ such that $\operatorname{dim}_{R} M=\operatorname{dim} R / \mathfrak{p}$. The following inequalities are clear

$$
\operatorname{dim}_{R} M+\operatorname{grade}_{R}(\mathfrak{p}) \leqslant \operatorname{dim} R / \mathfrak{p}+\text { ht } \mathfrak{p} \leqslant \operatorname{dim} R .
$$


Since cmd $R \leqslant 1$, using [5, (1.2.10)] we have:

$$
\operatorname{grade}_{R}(\mathfrak{p})=\inf \left\{\text { depth } R_{\mathfrak{q}} \mid \mathfrak{p} \subseteq \mathfrak{q}\right\}=\operatorname{depth} R_{\mathfrak{p}} .
$$

By our first claim since $\mathfrak{p} \in \operatorname{Ass}_{R}(M)$, F-grade ${ }_{R} M=\operatorname{depth} R_{\mathfrak{p}}=\operatorname{grade}_{R}(\mathfrak{p})$. By $(*)$ we have

$$
\operatorname{dim}_{R} M+\text { F-grade }{ }_{R} M \leqslant \operatorname{dim} R,
$$

which is the second equality. Since F- $\operatorname{grade}_{R} M=\operatorname{Gfd}_{R} M$, by [7] and [16, (3.19)], we get that depth $R-\operatorname{depth}_{R} M \leqslant \mathrm{~F}^{-\operatorname{grade}_{R}} M$. This completes the proof.

The following Example shows that the hypothesis of finiteness of depth is necessary.

ExAmple 3.8. Let $(R, \mathfrak{m})$ be a local domain with $\operatorname{dim} R>0$ and let $K$ be its fraction field. It is clear that $K$ is GF-perfect but depth ${ }_{R} K=\infty$ and $\operatorname{dim}_{R} K=\operatorname{dim} R$.

The following result is analogous to the Auslander-Bridger formula for the Gorenstein dimension [1]. We remark that, when $R$ is a Cohen-Macaulay local ring and $M$ is a finite $R$-module, it is clear that, $\operatorname{grade}_{R} M+\operatorname{dim}_{R} M=\operatorname{dim} R$. Viewing this, the following result is also an extension of this fact in the nonfinite case.

Corollary 3.9. Let $(R, \mathfrak{m})$ be a local Cohen-Macaulay ring and let $M$ be a GF-perfect module of finite depth. Then

$$
\operatorname{Gfd}_{R} M+\operatorname{depth}_{R} M=\operatorname{depth} R .
$$

Lemma 3.10. Let $R$ be a ring and let $x$ be an $R$ and $M$-regular element. Set $S=R / x R$. Then

$$
\operatorname{Rfd}_{R} M=\operatorname{Rfd}_{S}(M / x M) .
$$

Proof. Set $\bar{X}=X \otimes_{R} S$ for a module $X$. It is a simple computation that in the exact sequence

$$
0 \longrightarrow K \longrightarrow F \longrightarrow M \longrightarrow 0,
$$

when $F$ is a flat $R$-module we have $\operatorname{Rfd}_{R} K=0$, if $\operatorname{Rfd}_{R} M=0$ and if $\operatorname{Rfd}_{R} M>0$ then $\operatorname{Rfd}_{R} K=\operatorname{Rfd}_{R} M-1$. We will induct on $n=\operatorname{Rfd}_{R} M$. Let $L$ be a module such that $\mathrm{fd}_{S} L<\infty$, thus $\mathrm{fd}_{R} L<\infty$. By [17, page 140], we have $\operatorname{Tor}_{i}^{S}(L, \bar{M})=\operatorname{Tor}_{i}^{R}(L, M)$. So if $\operatorname{Rfd}_{R} M=0$ thus $\operatorname{Rfd}_{S} \bar{M}=0$. 
Now let $n>0$. Consider the exact sequence $0 \longrightarrow K \longrightarrow F \longrightarrow M \longrightarrow 0$, where $F$ is a free module. Since $x$ is both $F$ and $M$-regular, by [5, (1.1.5)] the following sequence is again exact

$$
0 \longrightarrow \bar{K} \longrightarrow \bar{F} \longrightarrow \bar{M} \longrightarrow 0 .
$$

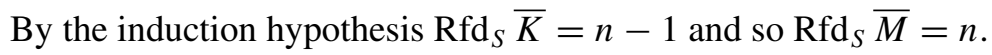

The following theorem is a generalization of a theorem due to Auslander and Bridger in [1] on the behavior of the Gorenstein dimension under base change.

THeOREM 3.11. Let $R$ be a ring and let $M$ be an $R$-module with $\operatorname{Gfd}_{R} M<$ $\infty$. Let $x$ be an $R$ and $M$-regular element, and let $S=R / x R$. Then

(1) $\operatorname{Gfd}_{R} M=\operatorname{Gfd}_{S}(M / x M)$.

(2) $\operatorname{Gfd}_{R}(M / x M)=\operatorname{Gfd}_{R} M+1$.

Proof. Set $\bar{X}=X \otimes_{R} S$ for a module $X$. For part (1) we argue by induction on $g=\operatorname{Gfd}_{R} M$. Let $g=0$, so $M$ is a Gorenstein flat $R$-module. Consider a complete resolution of flat modules as the following

$$
\cdots \longrightarrow F_{1} \stackrel{\partial_{1}}{\longrightarrow} F_{0} \stackrel{\partial_{0}}{\longrightarrow} F_{-1} \stackrel{\partial_{-1}}{\longrightarrow} F_{-2} \longrightarrow \cdots
$$

such that $M \simeq \operatorname{ker} \partial_{0}$; and for all $i \in \mathrm{Z}$ each $\operatorname{ker} \partial_{i}=M_{i}$ is a Gorenstein flat $R$-module and has the flat resolution as

$$
\cdots \longrightarrow F_{i+2} \longrightarrow F_{i+1} \longrightarrow M_{i} \longrightarrow 0 .
$$

Since $x$ is $M_{i}$-regular and $F_{i}$-regular for all $i$, by [5, (1.1.5)] the following sequence is again exact

$$
\cdots \longrightarrow \bar{F}_{i+2} \longrightarrow \bar{F}_{i+1} \longrightarrow \bar{M}_{i} \longrightarrow 0 .
$$

If we splice these sequences to each other we get a long exact sequence of flat $S$-modules

$$
\cdots \longrightarrow \bar{F}_{1} \stackrel{\bar{\partial}_{1}}{\longrightarrow} \bar{F}_{0} \stackrel{\bar{\partial}_{0}}{\longrightarrow} \bar{F}_{-1} \stackrel{\bar{\partial}_{-1}}{\longrightarrow} \bar{F}_{-2} \longrightarrow \cdots .
$$

Let $J$ be an injective $S$-module, hence by [17, page 140] we have

$$
\operatorname{Tor}_{\ell}^{S}\left(J, \bar{M}_{i}\right)=\operatorname{Tor}_{\ell}^{R}\left(J, M_{i}\right) \quad \text { for all } \ell \geqslant 0 .
$$

Since $\operatorname{id}_{R} J<\infty, \operatorname{Tor}_{\ell}^{R}\left(J, M_{i}\right)=0$ for $\ell>0$. So $(*)$ is a complete flat resolution of $S$-modules such that $\bar{M}=\operatorname{ker} \bar{\partial}_{0}$ and hence $\bar{M}$ is a Gorenstein flat $S$-module. 
Now let $g>0$, so there is a Gorenstein flat $R$-module $G$ and an $R$-module $L$ with $\operatorname{Gfd}_{R} L=g-1$, such that the following sequence is exact

$$
0 \longrightarrow L \longrightarrow G \longrightarrow M \longrightarrow 0 .
$$

Since $x$ is $G$-regular, by [5, (1.1.5)] the following sequence of $S$-modules is exact

$$
0 \longrightarrow \bar{L} \longrightarrow \bar{G} \longrightarrow \bar{M} \longrightarrow 0 .
$$

By the induction hypothesis, $\operatorname{Gfd}_{S} \bar{L}=g-1$ and $\operatorname{Gfd}_{S} \bar{G}=0$, therefore $\operatorname{Gfd}_{S} \bar{M}<\infty$. Now [16, (3.19)] and lemma (3.10) give the desired equality.

(2) Since $x$ is $M$-regular we have

$$
0 \longrightarrow M \stackrel{\cdot x}{\longrightarrow} M \longrightarrow \bar{M} \longrightarrow 0 .
$$

From [16] we have $\operatorname{Gfd}_{R} \bar{M}<\infty$, and by [16, (3.19)] and [22, (3.6)] we get $\operatorname{Gfd}_{R} \bar{M}=\operatorname{Rfd}_{R} \bar{M}=\operatorname{Rfd}_{S} \bar{M}+1$. But from Lemma (3.10) we find that $\operatorname{Gfd}_{S} \bar{M}=\operatorname{Rfd}_{S} \bar{M}=\operatorname{Gfd}_{R} M$ and this completes the proof of part (2).

Corollary 3.12. Let $R$ be $a$ ring and let $M$ be $a$ GF-perfect $R$-module. If $x$ is an $R$ and $M$-regular element and $S=R / x R$ and $\bar{M}=M / x M$, then $\bar{M}$ is a GF-perfect $S$-module.

Proof. It follows from Theorem (3.11) that $\operatorname{Gfd}_{S} \bar{M}<\infty$, and so F-grade $_{S} \bar{M}<\infty$. Let $n=\operatorname{Gfd}_{R} M=\mathrm{F}$-grade $R$ and $m=\mathrm{F}_{R} \operatorname{grade}_{S} \bar{M}$. Thus there is a flat $S$-module $F$, such that $\operatorname{Ext}_{S}^{m}(\bar{M}, F) \neq 0$. Since fd ${ }_{R} F<\infty$, using [17, page 140] it is not difficult to see that $n \leq m$. On the other hand, by Theorem (3.11), $n=\operatorname{Gfd}_{R} M=\operatorname{Gfd}_{S} \bar{M} \geq \mathrm{F}^{-\operatorname{grade}_{S}} \bar{M}=m$, and so $n=m$. Now it follows that $\bar{M}$ is a GF-perfect $S$-module.

ACKNOWLEDGEMENT. We are grateful to the referee for carefully reading the first version of this article. Parviz Sahandi would like to thanks his supervisor Professor Siamak Yassemi for using his grant from (IPM) (No. 84130216).

\section{REFERENCES}

1. Auslander, M., and Bridger, M., Stable module theory, Mem. Amer. Math. Soc. 94 (1969).

2. Auslander, M., and Buchsbaum, D., Homological dimension in noetherian rings. II, Trans. Amer. Math. Soc. 88 (1958) 194-206.

3. Bass, H., On the ubiquity of Gorenstein rings, Math. Z. 82 (1963), 8-28.

4. Brodmann, M., and Sharp, R., Local Cohomology: An Algebraic Introduction with Geometric Applications, Cambridge Stud. Adv. Math. 60 (1998).

5. Bruns, W., and Herzog, J., Cohen-Macaulay rings, Cambridge Stud. Adv. Math. 39 (1993).

6. Chouinard II, Leo G., On finite weak and injective dimension, Proc. Amer. Math. Soc. 60 (1976), 57-60. 
7. Christensen, L. W., Foxby, H. B., and Frankild, A., Restricted homological dimensions and Cohen-Macaulayness, J. Algebra 251 (2002), no. 1, 479-502.

8. Enochs, E. E., and Jenda, O. M. G., Gorenstein injective and projective modules, Math. Z. 220 (1995), no. 4, 611-633.

9. Enochs, E. E., Jenda, O. M. G., and Torrecillas, B., Gorenstein flat modules, Nanjing Daxue Xuebao Shuxue Bannian Kan 10 (1993), 1-9.

10. Enochs, E. E., and Jenda, O. M. G., Mock finitely generated Gorenstein injective modules and isolated singularities, J. Pure Appl. Algebra 96 (1994), 259-269.

11. Enochs, E. E., and Jenda, O. M. G., Gorenstein injective dimension and Tor-depth of modules, Arch. Math. (Basel) 72 (1999), 107-117.

12. Enochs, E. E., Flat covers and flat cotorsion modules, Proc. Amer. Math. Soc. 92 (1984), 179-184.

13. Foxby, H. B., Quasi-perfect modules over Cohen-Macaulay rings, Math. Nachr. 66 (1975), 103-110.

14. Foxby, H. B., n-Gorenstein rings, Proc. Amer. Math. Soc. 42 (1974), 67-72.

15. Foxby, H. B., Hyperhomological algebra and commutative rings, Københavns Univ. Mat. Inst., Preprint, 1998.

16. Holm, H., Gorenstein homological dimensions, J. Pure Appl. Algebra 189 (2004), 167-193.

17. Matsumura, H., Commutative Ring Theory, Cambridge Stud. Adv. Math. 8 (1986).

18. Rees, D., The grade of an ideal or module, Math. Proc. Cambridge Philos. Soc. 53 (1957), 28-42.

19. Roberts, P., Le théorème d'intersection, C. R. Acad. Sci. Paris Sér. I Math 304 (1987), 177180.

20. Sazeedeh, R., Gorenstein injective modules and local cohomology, Proc. Amer. Math. Soc. 132 (2004), no. 10, 2885-2891.

21. Sharif, T., and Yassemi, S., A generalization of Auslander-Buchsbaum and Bass formulas, Comm. Algebra 30 (2002), 869-875.

22. Sharif, T., and Yassemi, S., Depth formulas, restricted Tor-dimension under base change, Rocky Mountain J. Math. 34 (2004), 1131-1146.

23. Takahashi, R., The existence of finitely generated modules of finite Gorenstein injective dimension, Proc. Amer. Math. Soc. 134 (2006) no. 11, 3115-3121.

24. Xu, J., Flat Covers of Modules, Lecture Notes in Math. 1634 (1996).

25. Yassemi, S., A generalization of a theorem of Bass, Comm. Algebra 35 (2007) no. 1, 249-251.

DEPARTMENT OF MATHEMATICS

UNIVERSITY OF TEHRAN

TEHRAN

IRAN

E-mail: sahandi@ipm.ir
INSTITUTE FOR STUDIES IN THEORETICAL PHYSICS AND MATHEMATICS TEHRAN

IRAN

E-mail: sahandi@ipm.ir, sharif@ipm.ir 